\title{
CONSIDERATIONS IN FAVOR OF THE USE OF FISH OIL FOR CARDIOVASCULAR DISEASE PREVENTION AND TREATMENT IN OLDER ADULTS
}

\author{
I.M. Muo ${ }^{1}$, M. Miller', A.P. Goldberg ${ }^{1}$
}

\begin{abstract}
Omega-3 polyunsaturated fatty acids (fish oils) cause many metabolic benefits such as reductions in hypertriglyceridemia, blood pressure, markers of inflammation that may help to prevent or treat cardiovascular disease (CVD), particularly in older high risk patients. However, recent meta-analyses question these health benefits of fish oil supplementation. Studies show fish oil to be beneficial in cardiovascular health particularly when combined with physical activity and low cholesterol diet even though certain cardiovascular medications can interact with fish oil to affect its clinical response. In this review, we present clinical, behavioral and pharmacological factors such as age, genetics, gender, medications and lifestyle which can influence patients' biological responses to fish oil supplementation. We conclude that these factors, which are not typically accounted for in many clinical trials, may significantly contribute to the negative findings of these meta-analyses.
\end{abstract}

Key words: Omega 3 fatty acids, fish oil, cardiovascular disease, metabolic syndrome, aging.

\section{Introduction}

Numerous studies have evaluated the effectiveness of omega-3 polyunsaturated fatty acids (fish oil) for primary and secondary prevention of cardiovascular diseases (CVD) in older adults. Fish oils show promise in reducing cardiovascular disease risk due to their modulating effects on several mechanisms implicated in the pathogenesis of CVD. Fish oils reduce the adverse effects of free radicals, cytokines and other metabolites that injure the endothelial wall, cause foam cell formation, and vascular wall remodeling which contribute to atherosclerosis, metabolic syndrome and CVD mortality (Figure 1) (1, 2).

Fish oils are effective in reducing metabolic risk factors for CVD. In individuals with hypertriglyceridemia, fish oils can lower triglycerides (TG) by over $40 \%$ by enhancing free fatty acid oxidation (3). Fish oil also reduces markers of inflammation such as TNF-alpha, C-reactive protein, interleukin 6 to reduce vascular stress and reactivity. In obese, older adults with CVD risk factors, the combination of fish oil and weight loss

1. Division of Gerontology and Geriatric Medicine; 2. Division of Cardiovascular Medicine, University of Maryland School of Medicine, Baltimore, MD and Baltimore Geriatric Research, Education and Clinical Center, VA Maryland Health Care System, Baltimore, MD 21201

Corresponding Author: I.M. Muo, NICHD, National Institute of Health, 10 Center Drive, Bldg 10, CRC, Room 1-3140, MSC 1109, Bethesda, MD 20814, Email: imuo@ grecc.umaryland.edu reduces metabolic risk factors and improves vascular compliance more than with weight loss alone (4). Fish oils may also help to prevent sarcopenia, reduce diabetes incidence, boost physical and cognitive functions and reduce depressive symptoms in older adults (5-9). These pleiotropic effects can translate globally into healthier aging through improved cardiovascular, musculoskeletal and neurologic health. Yet, recent meta-analyses question the cardio-protective effects of fish oils in high-risk older adults $(10,11)$. In this review, we review known-patient and medication factors that may affect the clinical response of fish oils for prevention and treatment of CVD.

\section{Omega 3-Polyunsaturated Fatty Acid: Sources, Metabolism and Pharmacology}

\section{Sources and Metabolism}

Omega 3 (n-3 PUFA) is one of the two known types of polyunsaturated fatty acids-the other is Omega 6 (n-6 PUFA) polyunsaturated fatty acids. Both are essential fatty acids for humans, thus they must be consumed in the diet or as nutri-supplements. Both PUFAs are long chain fatty acids whose metabolism start in the cell membrane and continue in the peroxisomes by the same enzymes (12). n-3 PUFA differs from omega-6 polyunsaturated fatty acids by the location of the first 
double bond. Alpha linoleic acid (ALA), eicosapentaenoic acid (EPA) and docosahexaenoic acid (DHA) are three major known dietary sources of n-3 PUFA. Fish and fish oil supplements are the reliable sources of dietary DHA and EPA because ALA is metabolized, though very inefficiently, to EPA and DHA. Metabolites of omega-3 PUFA are largely anti-inflammatory while metabolites of n-6 PUFA, such as arachidonic acid, are proinflammatory when compared to n-3 PUFA metabolites. Dietary sources of 1) n-3 PUFA include salmon, pollock, herring, albacore tuna, 2) ALA include walnuts, flaxseed, soybeans and canola oils and 3) n-6 PUFA include safflower, sunflower, corn, soybean oils (12).

\section{Absorption and distribution of fish oils}

Most fish oil supplements are available as over-thecounter nutraceuticals. The absorption and plasma levels of DHA and EPA differ based on formulation. Emulsified fish oils are digested and absorbed faster than encapsulated fish oil (13). Studies conducted in healthy adults show that re-esterified lipid formulations of fish oil have higher n-3 PUFA levels in red blood cell phospholipids than do ethyl ester formulations (14). Further research is needed to determine the effects of EPA and DHA formulation on absorption and CVD risk factors in older adults.
The American Heart Association (AHA) recommends daily intake of $1 \mathrm{~g} \mathrm{EPA}+\mathrm{DHA}$ for individuals with coronary artery disease and higher doses (2-4g of combined EPA, DHA) in individuals with hypertriglyceridemia $(15,16)$. Recently, some investigators have called for revision of these currently recommended daily intakes for omega 3 fatty acids (17). The doses of EPA and DHA in diet and fish oil supplements may affect the degree of and rapidity of their primary and secondary prevention of CVD. Depending on the dosages, the levels of EPA and DHA distribute differently into various cell membranes such as red blood cells, leukocytes, platelets. Furthermore, the rate of saturation of omega- 3 fatty acids into these various cell membranes over time depends heavily on the type of cell (18). Within each particular cell group, higher dosing of fish oils leads to faster incorporation; however, the time to maximal incorporation varies across cell types. For instance, full incorporation of EPA or DHA into platelets takes 20 to 30-days, while in lymphocytes it takes 6 to 8 -months (18). Since the chronic inflammatory process in atherosclerosis involves various cell types, one can only speculate that the dose and duration of fish oil therapy and patient's age influence the observed cardiovascular clinical outcomes by affecting the biological cascades resulting from n-3 PUFA incorporation into the different cell membranes.

\section{Figure 1}

Potential Sites of Fish Oil Modification of Changes that Occur with Aging

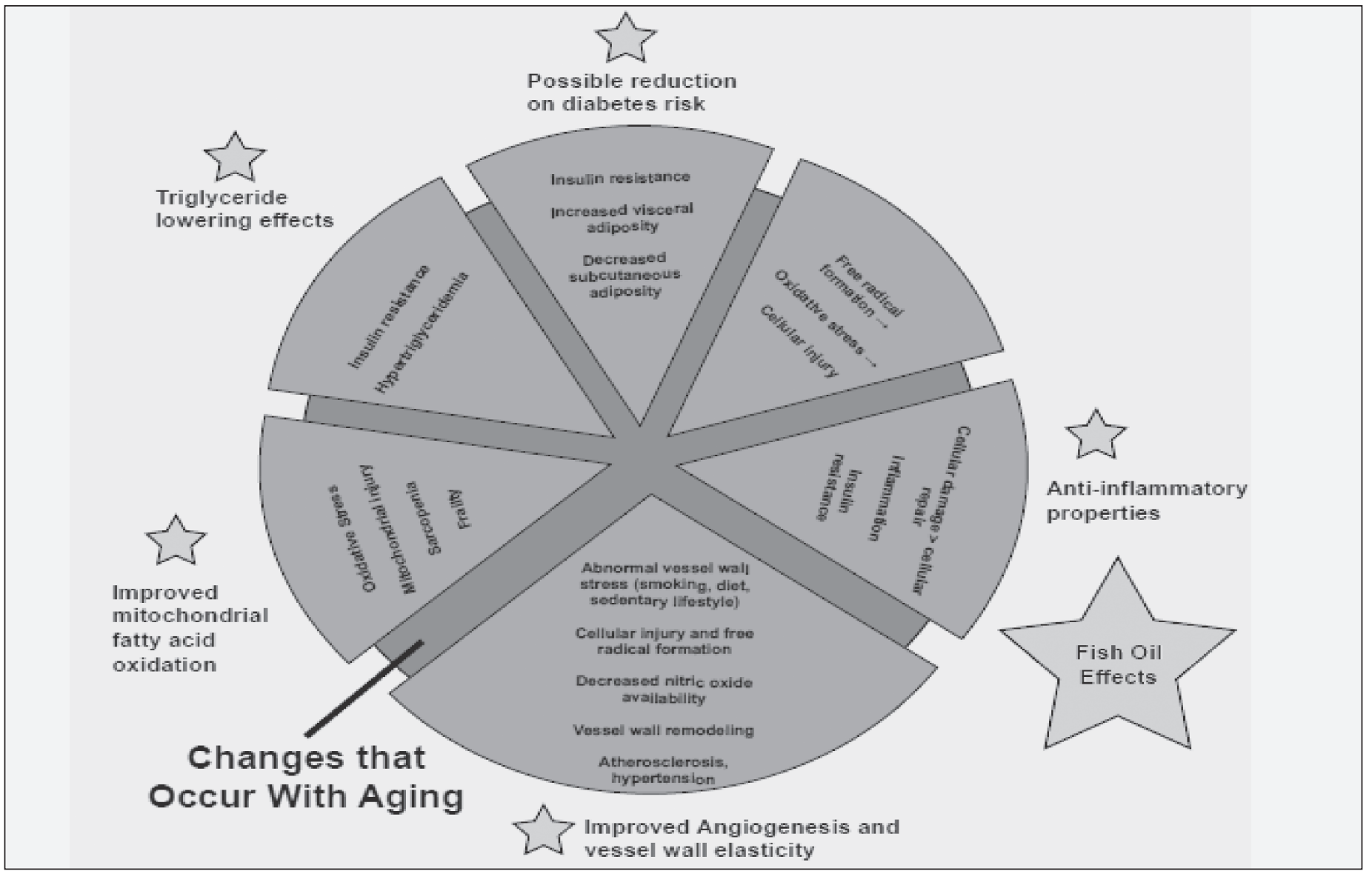


Thus, it is possible that the relatively low dosage of $1-2 \mathrm{~g} /$ day of fish oil supplements administered to patients at high CVD risk in many of the studies referenced in the meta-analyses may explain the poor clinical responses.

\section{Side effects and drug-interactions}

A major concern with too much fish and fish oil consumption is toxicity with organic pollutants such as methyl-mercury, polychlorinated biphenyls, organochlorines, polyaromatic hydrocarbons and dioxins $(19,22,23)$. One multicenter, case-controlled study found that high levels of plasma methyl-mercury masked the cardio-protective effects of DHA $(20,21)$. As dietary supplements, most fish oil formulations are unregulated by the Food and Drug Administration. In the over-thecounter supplements studied, mercury and methylmercury were found to be within "negligible" levels $(<12 \mathrm{ng} / \mathrm{ml})$, even with consumption of high doses of the supplements. While these levels may apply to the general public, it is unknown what effects they may have in frail older adults with multiple disease co-morbidities.

Several studies also evaluate the interaction between fish oils and medications used to treat or prevent CVD, such as aspirin, statins and other anticoagulants such as Plavix and warfarin-medications which are frequently used in older adults with cardiovascular diseases. These studies show no life-threatening drug-drug interactions from using fish oils in combination with these cardiac medications. Instead, fish oils seem to provide complementary and even in some cases synergistic effects with these other cardiovascular treatments (24-26). For example, fish oils are shown to improve the anti-platelet effects of aspirin when used in combination with aspirin in individuals with $\mathrm{CAD}$, including those with evidence of aspirin resistance (25).

\section{Clinical Factors affecting response to fish oil supplements}

\section{Age, genetics and gender}

The level and effects of EPA and DHA appear to differ by gender and age. Younger women tend to have higher DHA levels than men due to higher 17-beta estradiol, which seems to mediate this gender difference in DHA by increasing hepatic production of DHA. In contrast, EPA levels do not differ by gender $(27,28)$. Men and women also respond differently to DHA and EPA. In men, EPA has a greater antiplatelet effect than DHA, whereas the reverse is true in women (29). Even so, EPA may provide other vascular protective effects in women. Low levels of EPA in women are associated with increased mortality from all causes after acute myocardial infarction (30). Genetic variation in several enzymes involved in the metabolism of PUFA such as delta 5 and delta 6 desaturases, cyclooxygenase and lipooxygenase have significant independent effects on the risk for cardiovascular events $(31,32)$. The prevalence of these age, gender and genetic variations in response to fish oil preparations and their clinical utility should be considered in future clinical research studies.

\section{Diet, exercise and CVD risk, interactions with fish oils}

Diet remains highly relevant in CVD prevention and reduction of morbidity and mortality with aging (33). The Mediterranean diet, which is rich in vegetables, nuts, fish, and legumes, was recently shown in a large prospective study to decrease the risk of cardiovascular events among high cardiovascular risk, dietary compliant individuals (34). Compliance with the AHA's recommended dietary guidelines improve lipid profiles in overweight and obese older adults-especially when combined with weight loss $(35,36)$. Moreover, a cross sectional study in middle-aged adults suggests that the beneficial effects of fish oil in reducing cardiometabolic risk may be present only in individuals with high baseline physical activity (37). Thus, future fish oil studies should consider the participants' prior diet and physical activity habits when evaluating clinical outcomes of fish oil supplementation.

\section{Summary and future considerations}

Cardiovascular disease is the leading cause of mortality in the United States, and age represents a significant variable in deriving 10-year risk, using the Framingham Risk Score. Patients with coronary artery

Table 1

Caveats with using fish oil supplements for cardiovascular disease prevention and treatment

\footnotetext{
Patient factors 1. No guidelines as to the optimal time/age to start fish oil for primary and secondary CVD prevention. 2. Limited understanding of the effects of age, genetics and gender on fish oil pharmacology and on clinical outcomes.

3. Little knowledge of the effects of co-morbidities on fish oil pharmacology

Fish oil factors 1. Un-prescribed fish oil supplements are not FDA regulated.

2. Potential for adverse health risks of contaminants in fish and over-the-counter fish oil supplements.

3. Unknown levels of toxic contaminants in many over-the-counter fish oil supplements.
} 
disease (CAD) and hypertriglyceridemia (e.g., TG > 150 $\mathrm{mg} / \mathrm{dL}$ ) remain at increased risk of future CAD events, despite attainment of their LDL treatment goal with statin therapy (38). Yet, despite elevated risk associated with high TG levels in middle and older-aged adults, it is not known whether lowering TGs translates into reduced CVD risk. Currently, the REDUCE-IT trial (clinicaltrials. gov) is evaluating whether a purified EPA derivative, (icosapent ethyl) reduces CVD events beyond standard of care therapy in hypertriglyceridemic patients with or at increased CVD risk. This study is expected to be completed in 2016.

Aging is also associated with changes in body composition, with a predilection for an increase in visceral fat mass and a loss of muscle mass. Fish oils show promising role in preventing or slowing sarcopenia. It is not known whether reducing visceral adiposity in older adults with chronic fish oil supplementation leads to an improvement in cardio-metabolic risk factors and to a reduction in insulin resistance. Research is underway in our laboratory to evaluate the potential of chronic fish oil supplementation to reduce visceral fat accumulation in subjects with metabolic syndrome. Such a finding could have major public health implications.

While the role of fish oils in preventing CVD and CVD mortality remains to be fully established, fish oil supplements are generally well tolerated by older people. Side effects of fish oil supplements are minimal and include symptoms such as bloating and diarrhea, which are dose dependent. At the same time, most available fish oil supplements are from over-the-counter and the levels of toxic substances in them are variable (Table 1). The benefits of fish oil supplementation outweigh the risks due to the pleiotropic effects of fish oils beyond the cardiovascular system These benefits warrant continued research on the actions of fish oils in various clinical phenotypes such as metabolic syndrome, hypertriglyceridemia and cardiovascular disease (Figure 1). Both medication, age and patient-related clinical factors can modify the responses and long-term value of fish oil supplementation for primary and secondary CVD prevention. No studies are known to date which have evaluated the aggregate clinical effects of each of these modifying factors. Patients who wish to take fish oil supplements for cardiovascular risk reduction can optimize the clinical effects of fish oils by following a heart healthy diet and participating in regular physical activity.

Acknowledgements: This work was supported by funds from: National Institute on Aging (NIA) grants: 5T32AG000219-19, Claude D. Pepper Older Americans Independence Center (P30 AG028747), NIDDK Mid-Atlantic Nutrition Obesity Research Center (NIH P30 DK072488); Department of Veterans Affairs and Veterans Affairs Medical Center Baltimore Geriatric Research, Education and Clinical Center (GRECC). Special thanks to Seth Crawford of the Baltimore VA Medical Media Service.

Conflict of interest: Author MM is a consultant and steering committee member of REDUCE-IT trial for Amarin.

\section{References}

1. Hansson GK. Inflammation, atherosclerosis, and coronary artery disease. N Engl J Med. 2005;352(16):1685-1695

2. Lakka HM, Laaksonen DE, Lakka TA, et al. The metabolic syndrome and total and cardiovascular disease mortality in middle-aged men. JAMA. 2002;288(21):2709-2716.

3. Phillipson BE, Rothrock DW, Connor WE, Harris WS, Illingworth DR. Reduction of plasma lipids, lipoproteins, and apoproteins by dietary fish oils in patients with hypertriglyceridemia. N Engl J Med. 1985;312(19):1210-1216.

4. Wong AT, Chan DC, Barrett PH, Adams LA, Watts GF. Supplementation with $\mathrm{n} 3$ fatty acid ethyl esters increases large and small artery elasticity in obese adults on a weight loss diet. J Nutr. 2013;143(4):437-441.

5. Sinn N, Milte CM, Street SJ, et al. Effects of n-3 fatty acids, EPA v. DHA, on depressive symptoms, quality of life, memory and executive function in older adults with mild cognitive impairment: A 6-month randomised controlled trial. Br J Nutr. 2012;107(11):1682-1693.

6. Rondanelli M, Giacosa A, Opizzi A, et al. Effect of omega-3 fatty acids supplementation on depressive symptoms and on health-related quality of life in the treatment of elderly women with depression: A double-blind, placebo-controlled, randomized clinical trial. J Am Coll Nutr. 2010;29(1):5564 .

7. Smith GI, Atherton P, Reeds DN, et al. Dietary omega-3 fatty acid supplementation increases the rate of muscle protein synthesis in older adults: A randomized controlled trial. Am J Clin Nutr. 2011;93(2):402-412.

8. Rodacki CL, Rodacki AL, Pereira G, et al. Fish-oil supplementation enhances the effects of strength training in elderly women. Am J Clin Nutr. 2012;95(2):428-436.

9. Djousse L, Gaziano JM, Buring JE, Lee IM. Dietary omega-3 fatty acids and fish consumption and risk of type 2 diabetes. Am J Clin Nutr. 2011;93(1):143150

10. Rizos EC, Ntzani EE, Bika E, Kostapanos MS, Elisaf MS. Association between omega-3 fatty acid supplementation and risk of major cardiovascular disease events: A systematic review and meta-analysis. JAMA. 2012;308(10):10241033.

11. Kwak SM, Myung SK, Lee YJ, Seo HG, Korean Meta-analysis Study Group. Efficacy of omega-3 fatty acid supplements (eicosapentaenoic acid and docosahexaenoic acid) in the secondary prevention of cardiovascular disease: A meta-analysis of randomized, double-blind, placebo-controlled trials. Arch Intern Med. 2012;172(9):686-694.

12. De Caterina R. N-3 fatty acids in cardiovascular disease. N Engl J Med. 2011;364(25):2439-2450.

13. Raatz SK, Redmon JB, Wimmergren N, Donadio JV, Bibus DM. Enhanced absorption of n-3 fatty acids from emulsified compared with encapsulated fish oil. J Am Diet Assoc. 2009;109(6):1076-1081.

14. Neubronner J, Schuchardt JP, Kressel G, Merkel M, von Schacky C, Hahn A. Enhanced increase of omega-3 index in response to long-term n-3 fatty acid supplementation from triacylglycerides versus ethyl esters. Eur J Clin Nutr. 2011;65(2):247-254.

15. Krauss RM, Eckel RH, Howard B, et al. AHA dietary guidelines: Revision 2000: A statement for healthcare professionals from the nutrition committee of the american heart association. Circulation. 2000;102(18):2284-2299.

16. Kris-Etherton PM, Harris WS, Appel LJ, AHA Nutrition Committee. American Heart Association. Omega-3 fatty acids and cardiovascular disease: New recommendations from the american heart association. Arterioscler Thromb Vasc Biol. 2003;23(2):151-152.

17. Flock MR, Harris WS, Kris-Etherton PM. Long-chain omega-3 fatty acids: Time to establish a dietary reference intake. Nutr Rev. 2013;71(10):692-707.

18. Browning LM, Walker CG, Mander AP, et al. Incorporation of eicosapentaenoic and docosahexaenoic acids into lipid pools when given as supplements providing doses equivalent to typical intakes of oily fish. Am J Clin Nutr. 2012;96(4):748-758.

19. Melanson SF, Lewandrowski EL, Flood JG, Lewandrowski KB. Measurement of organochlorines in commercial over-the-counter fish oil preparations: Implications for dietary and therapeutic recommendations for omega-3 fatty acids and a review of the literature. Arch Pathol Lab Med. 2005;129(1):74-77.

20. Guallar E, Sanz-Gallardo MI, van't Veer P, et al. Mercury, fish oils, and the risk of myocardial infarction. N Engl J Med. 2002;347(22):1747-1754.

21. Wennberg M, Stromberg U, Bergdahl IA, et al. Myocardial infarction in relation to mercury and fatty acids from fish: A risk-benefit analysis based on pooled finnish and swedish data in men. Am J Clin Nutr. 2012;96(4):706-713.

22. Drabova L, Pulkrabova J, Kalachova K, Tomaniova M, Kocourek V, Hajslova J. Polycyclic aromatic hydrocarbons and halogenated persistent organic pollutants in canned fish and seafood products: Smoked versus non-smoked products. Food Addit Contam Part A Chem Anal Control Expo Risk Assess. 2013;30(3):515-527.

23. Ashley JT, Ward JS, Anderson CS, et al. Children's daily exposure to polychlorinated biphenyls from dietary supplements containing fish oils. Food Addit Contam Part A Chem Anal Control Expo Risk Assess. 2013;30(3):506-514.

24. Harris WS. Expert opinion: Omega-3 fatty acids and bleeding-cause for 
concern? Am J Cardiol. 2007;99(6A):44C-46C.

25. Lev EI, Solodky A, Harel N, et al. Treatment of aspirin-resistant patients with omega-3 fatty acids versus aspirin dose escalation. J Am Coll Cardiol. 2010;55(2):114-121.

26. Doenyas-Barak K, Berman S, Abu-Hamad R, Golik A, Rahimi-Levene N, Efrati S. N-3 fatty acid supplementation to routine statin treatment inhibits platelet function, decreases patients' daytime blood pressure, and improves inflammatory status. Eur J Clin Pharmacol. 2012;68(8):1139-1146.

27. Giltay EJ, Gooren LJ, Toorians AW, Katan MB, Zock PL. Docosahexaenoic acid concentrations are higher in women than in men because of estrogenic effects. Am J Clin Nutr. 2004;80(5):1167-1174.

28. Giltay EJ, Duschek EJ, Katan MB, Zock PL, Neele SJ, Netelenbos JC. Raloxifene and hormone replacement therapy increase arachidonic acid and docosahexaenoic acid levels in postmenopausal women. J Endocrinol. 2004;182(3):399-408.

29. Phang M, Lincz LF, Garg ML. Eicosapentaenoic and docosahexaenoic acid supplementations reduce platelet aggregation and hemostatic markers differentially in men and women. J Nutr. 2013;143(4):457-463.

30. Lee SH, Shin MJ, Kim JS, et al. Blood eicosapentaenoic acid and docosahexaenoic acid as predictors of all-cause mortality in patients with acute myocardial infarction--data from infarction prognosis study (IPS) registry. Circ J. 2009;73(12):2250-2257.

31. Lu Y, Vaarhorst A, Merry AH, et al. Markers of endogenous desaturase activity and risk of coronary heart disease in the CAREMA cohort study.
PLoS One. 2012;7(7):e41681.

32. Simopoulos AP. Genetic variants in the metabolism of omega- 6 and omega- 3 fatty acids: Their role in the determination of nutritional requirements and chronic disease risk. Exp Biol Med (Maywood). 2010;235(7):785-795.

33. Samieri C, Sun Q, Townsend MK, et al. The association between dietary patterns at midlife and health in aging: An observational study. Ann Intern Med. 2013;159(9):584-591.

34. Estruch R, Ros E, Martinez-Gonzalez MA. Mediterranean diet for primary prevention of cardiovascular disease. N Engl J Med. 2013;369(7):676-677.

35. Dengel JL, Katzel LI, Goldberg AP. Effect of an american heart association diet, with or without weight loss, on lipids in obese middle-aged and older men. Am J Clin Nutr. 1995;62(4):715-721.

36. Nicklas BJ, Katzel LI, Bunyard LB, Dennis KE, Goldberg AP. Effects of an american heart association diet and weight loss on lipoprotein lipids in obese, postmenopausal women. Am J Clin Nutr. 1997;66(4):853-859.

37. Muldoon MF, Erickson KI, Goodpaster BH, et al. Concurrent physical activity modifies the association between $\mathrm{n} 3$ long-chain fatty acids and cardiometabolic risk in midlife adults. J Nutr. 2013;143(9):1414-1420.

38. Miller M, Cannon CP, Murphy SA, et al. Impact of triglyceride levels beyond low-density lipoprotein cholesterol after acute coronary syndrome in the PROVE IT-TIMI 22 trial. J Am Coll Cardiol. 2008;51(7):724-730. 